\title{
sciendo
}

\author{
Current Issues in Pharmacy and Medical Sciences \\ Formerly ANNALES UNIVERSITATIS MARIAE CURIE-SKLODOWSKA, SECTIO DDD, PHARMACIA \\ journal homepage: http://www.curipms.umlub.pl/
}

\section{The role of Toll-like receptors (TLRs) in virus-related cancers: a mini review}

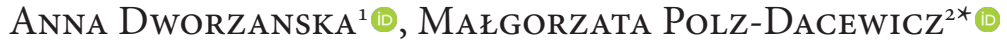

${ }^{1}$ Masovian Specialist Hospital, 26-617 Radom, Poland

${ }^{2}$ Department of Virology, Medical University of Lublin, 20-059 Lublin, Poland

\begin{tabular}{|c|c|}
\hline ARTICLE INFO & ABSTRACT \\
\hline $\begin{array}{l}\text { Received } 21 \text { February } 2020 \\
\text { Accepted } 16 \text { July } 2020\end{array}$ & $\begin{array}{l}\text { The modulation of the host innate immune system is a well-established carcinogenesis } \\
\text { feature of several tumors, including human Epstein-Barr (EBV) and Papillomavirus- }\end{array}$ \\
\hline $\begin{array}{l}\text { Keywords: } \\
\text { TLR9, } \\
\text { EBV, } \\
\text { HPV, } \\
\text { nasopharyngeal cancer, } \\
\text { gastric cancer. }\end{array}$ & $\begin{array}{l}\text { (HPV) related cancers. These viruses are able to interrupt the initial events of the } \\
\text { immune response, including the expression of Toll-like receptors (TLRs), cytokines, and } \\
\text { inflammation. The aim of the study is to review current data and summarize knowledge } \\
\text { on the TLRs and their role in the development of cancer, especially viral-related cancers } \\
\text { (EBV and HPV). Research work shows a correlation between the TLRs polymorphism } \\
\text { and the development of oropharyngeal and gastric cancer (GC), especially related to } \\
\text { viral infections. Many studies suggest the important role for TLRs in inflammatory, } \\
\text { autoimmune disease and human cancers. However, further efforts are necessary to draw } \\
\text { a precise conclusion. }\end{array}$ \\
\hline
\end{tabular}

\section{CHARACTERISTICS OF TOLL LIKE RECEPTORS}

Congenital immunity is the first line of an organism's defense against infection [1]. Its effect is determined by evolutionarily conserved molecular pattern recognition receptors (PRRs) that recognize different structures of microorganisms called PAMP (pathogen associated molecular patterns). Thanks to PPR, the human organism is capable if distinguishing foreign from its own antigens. PRR representatives include Toll-like receptors (TLRs), which play the major role in recognizing infection and triggering immunological response [2].

These receptors were discovered in Drospohila melanogaster. The PRRs are the mammalian homologue of the drosophila toll protein [2]. Till now, 13 TLRs has been described in mammals (10 receptors in humans and 12 in mice). The molecular structure of TLRs is comprised of two domains: the extracellular N-terminal containing leucinerich repeats responsible for PAMPs recognition, and the intracellular C-terminal domains that, by using conserved regions, transmit the signal for adapter molecules.

TLR transmembrane proteins are expressed by different subsets of immune and non-immune cell types, such as monocytes, macrophages, dendritic cells, neutrophils, B cells, T cells, fibroblasts, endothelial cells and epithelial cells, and play an essential role in innate and adaptive

\footnotetext{
* Corresponding author

e-mail:m.polz@umlub.pl
}

immunity against pathogenic agents such as bacteria and viruses [3]. Some TLRs reside at the plasma membrane, where they recognize molecular components located on the surface of pathogens. In contrast, others exist intracellularly, where they mediate recognition of nucleic acids. TLRs localized on the cell's surface interact with bacterial structures (TLRs 1, 2, 4, 5, 6) while those localized in the endosomes $(3,7,8,9)$ recognize viral nucleic acids (DNA and RNA).

Different PRRs react with specific PAMPs, leading to distinct expression patterns, specific signaling pathways, and distinct anti-pathogen responses. There are two major TLR pathways; one is mediated by myeloid differentiation primary response 88 (MyD88) adaptor proteins, and the other is independent to MyD88 [4]. With the exception of TLR3, all other TLRs commonly use MyD88 as the downstream adopter protein. Generally, upon activation with their individual ligands, activated TLRs recruit MyD88, leading to subsequent initiation of the downstream targets. These include a nuclear factor of kappa light polypeptide gene enhancer in B-cells (NF- $\mathrm{B}$ ), mitogen-associated protein (MAP) kinase and interferon regulatory factors (IRFs).

Recently, many studies have revealed that TLRs play a key role in the homeostasis of the human immune system. However, abnormal activation of TLRs may contribute to various diseases. Nowadays, more and more significant evidence suggested that TLRs play an important role in inflammatory, autoimmune disease and human cancers. 


\section{TLRS IN CANCERS}

According to modern theory, inflammation is the main agent causing the development and progression of cancer [5]. The causative mechanism of this process is not well understood. The cells in the inflammation site stimulate activity of the transcription factor NF- $\kappa \mathrm{B}$, which is responsible for the expression of anti-inflammatory cytokines and is a major antiapoptotic factor activating the expression of various genes. The interaction between highly conserved molecular domains of pathogens and TLRs play a pivotal role in oncogenesis. On one hand, TLRs can suppress tumor growth, on the other hand, they can stimulate cancer progression and influence cancer resistance to chemotherapy.

Meta-analysis has shown that TLR4 and TLR7 expression is significantly associated with poor survival in patients with various types of carcinoma [5]. Indeed, a number of dependencies were described between TLRs polymorphism on the one hand, and infectious diseases and cancer on the other [2]. Therefore, several TLRs were investigated in clinical prognosis studies and the results for a special TLR were inconsistent.

\section{TLRS IN EBV- AND HPV-RELATED CANCERS}

TLRs are expressed not only in immune cells, but also in the tumor microenvironment (TME). This is a complex arrangement of cancer cells, normal cells, stromal tissue and extracellular cytokine [6]. Viruses are intracellular pathogens that reproduce only in the living cell using the cellular machinery to produce new viral particles. Viral infection causes chronic inflammation leading to cell death, uncontrollable proliferation and modulated expression of some of the regulatory proteins. Approximately, $20 \%$ of all human oncogenesis are caused by cancer-causing viruses (oncoviruses). Oncogenic viruses encode genes that cause viral replication and transformation of the host cells to produce viral proteins and protein complexes. The phenomenon from basic viral infection to tumorigenesis is lengthy due to the involvement of factors like immunity complications, cellular mutations and exposure to other cancerous agents.

Epstein-Barr virus (EBV) and human papillomavirus (HPV), which are DNA viruses, are reported to be linked with $38 \%$ of all virus-associated cancers [7]. In cancers with an infective etiology, such as EBV and HPV, TLR responses may be activated and play a crucial role in tumorigenesis. Changes in the expression of TLRs or their signaling pathways may lead to progression or regression of the tumor, depending on type of cancer. Individual studies are, hence, necessary to assess the role of TLRs in a given cancer type, and the results cannot be predicted to other tumors. Therefore, the following section presents the results of the studies on the role of TLRs in nasopharyngeal (associated with EBV and/or HPV infection) and gastric cancer associated with EBV infection.

The EBV is a ubiquitous gammaherpesvirus that infects more than $90 \%$ of the global adult human population [7]. EBV infection is associated with various cancers, among others, nasopharyngeal carcinoma (NPC) and gastric cancer (GC). After primary infection, EBV establishes latent infection in memory B lymphocytes with periodic reactivation.

NPC is a highly invasive malignant tumour arising from the mucosal epithelium of the nasopharynx. NPC has marked geographical disparities in incidence, with the highest occurrence in Southeast Asia and lowest in Europe and North America [8].

TLR9 are capable of recognizing EBV and launching innate immune responses, which may limit the spread of the virus and may contribute to controlling the outgrowth of latently EBV-infected B cells [9]. On the other hand, EBV may interfere with the expression and functionality of TLR9, thereby manipulating host immune responses towards favoring long-term survival of the virus. TLR3 and TLR 9 are involved in detecting EBV and can complement each other in recognizing EBV. Both TLR3 and TLR 9 are secreted by lymphocytes $B$, which are the primary target for EBV. Latent membrane protein 1 (LMP1), a major oncoprotein of EBV, might inhibit TLR9 expression. As shown in studies by Tsao et al. [10], increased expression of LMP1 in B cells impeded the activity of TLR9 promoter, mRNA and the level of proteins. LMP mutants, on the other hand, show a changed ability to activate the NF-kB pathway, preventing dysregulation of TLR9 [11].

Our own studies showed that TLR9 concentration was statistically lower in patients with EBV-positive than EBVnegative GC (both in serum and tissue), with the level of TLR9 in tissue being statistically higher than in serum. Furthermore, the presence of early antigen (EA) brought about the lowering of the TLR9 levels, which may point to the role of TLR9 in the reactivation of EBV infection.

A strong association between the presence of HPV DNA and various cancers has been described, specifically HPV16, and Oral Squamous Cell Carcinoma (OSCC) [8], and many studies have demonstrated that HPV significantly increases the risk for OSCC, as compared with controls. Tobouti et al. [12] studied the expression of all TLRs in Oropharyngeal Squamous Cell Carcinoma (OPSCC) cell lines (both HPV positive and HPV negative). Results showed lower TLR1 and TLR6 mRNA expression and higher TLR9 protein expression in HPV-positive than in HPV negative OPSCC cells.

Although it is commonly accepted that EBV and HPV can contribute to carcinogenesis, these viruses alone seem insufficient for malignant transformation [13]. Ruuskanen et al. [14] demonstrated that TLRs were highly expressed in NPC, but the expression patterns of TLR2 and TLR5 were related to the viral status, while both TLRs were expressed significantly less in EBV-positive than in HPV-positive or EBV/HPV-negative NPC. Moreover, the authors suggest that TLR7 seems to be an independent prognostic factor for non-endemic NPC. In Squamous Cell Carcinoma (SCC) specimens, other researchers demonstrated that the TLRs 3, 4 , and 5 were significantly under-expressed, while TLR1 was significantly over-expressed (TLRs 2, 7, 8, and 9 were not significant) when compared to normal samples [15]. However, the opposite results were also observed [16].

Co-infection of EBV and HPV is usually found in various cancers, including: NPC, cervical, prostate, breast, gastric, lung and Esophageal Squamous Cell Carcinoma (ESCC) 
[19]. Here, genetic polymorphism of TLR 9 and TLR 4 may influence the effects of HPV/EBV, as well as co-infection, and play a significant role in development of the disease [17]. Modulation of TLR9 levels probably occurs due to HPV16 E7 oncoprotein activity on the TLR9 promoter by interference in the NF- $\kappa$ B pathway [18].

Recently, many biomarkers have been applied in cancer therapy diagnosis and monitoring. For example, TLRs may be used as biomarkers in virus-associated cancers. The modulation of the host innate immune system is also a well-established carcinogenesis feature of several tumors, including virus- related cancers (e.g. HPV or EBV). These viruses are able to interrupt the initial events of the immune response, including the expression of TLRs. Therefore, the imbalance of sensitive control of the immune response is an important factor for developing immunotherapies. Herein, the acting of TLR agonists as immunological adjuvants for anticancer immunotherapy has been extensively investigated over the past few years [20]. Co-infection has opened a new field with potential targets for the development of novel antiviral therapies. However, further studies are necessary to understand the relationship between TLR gene polymorphisms and EBVaGC, as well as EBV- and HPV-related NPC.

\section{FUNDING}

This study was supported by a Research Grant from the Medical University of Lublin, Poland (DS 233).

\section{ACKNOWLEDGMENTS}

We are thankful to Dr. Ewa Kliszczewska for technical preparation of manuscript.

\section{ORCID iDs}

Anna Dworzańska (Dhttps://orcid.org/0000-0001-7935-6736 Małgorzata Polz-Dacewicz

(1) https://orcid.org/0000-0002-3222-184X

\section{REFERENCE}

1. Kawai T, Akira S. The role of pattern-recognition receptors in innate immunity: update on Toll-like receptors. Nat Immunol. 2010;11(5): 373384.

2. Loganathan R, Nazeer M, Goda V, Devaraju P, Ali M, Karunakaran $P$, et al. Genetic variants of TLR4 and TLR9 are risk factors for chronic Helicobacter pylori infection in South Indian tamils. Hun Immunoy. 2017;78(2):216220.
3. Iwasaki A, Medzhitov R. Toll-like receptor control of the adaptive immune responses. Nat Immunol. 2004;5(10):987995.

4. So WY and Ouchi T. The application of Toll like receptors for cancer therapy. Int J Biol Sci. 2010;6(7):675681.

5. Wang Q, Zhang X, Xiao T, Pan C, Liu X, Zhao Y. Prognostic role of Toll-like receptors in cancer: a meta-analysis. Ther Clin Risk Manag. 2018;14:1323330.

6. González-Reyes S, Marín L, González L, González LO, del Casar JM, Lamelas ML, et al. Study of TLR3, TLR4 and TLR9 in breast carcinomas and their association with metastasis. BMC Cancer. 2010;10:665.

7. Mesri EA, Feitelson MA, Munger K. Human viral oncogenesis: a cancer hallmarks analysis. Cell Host Microbe. 2014;15(3):266-82.

8. Jemal A, Bray F, Center MM, Ferlay J, Ward E, Forman D. Global cancer statistics. CA Cancer J Clin. 2011;61(2):69-90.

9. Zauner L, Nadal D. Understanding TLR9 action in Epstein-Barr virus infection. Front Biosci (Landmark Ed).2012;17:1219-31.

10. Tsao SW, Tsang CM, To KF, Lo KW. The role of Epstein-Barr virus in epithelial malignancies. J Pathol. 2015;235(2):323333.

11. Liu S, Wang X, Shi Y, Han L, Zhao Z, Zhao Cd at al. Toll-like receptor gene polymorphisms and susceptibility to Epstein-Barr virus-associated and -negative gastric carcinoma in Northern China. Saudi J Gastroenterol. 2015;21(2):95-103.

12. Tobouti PL, Bolt R, Radhakrishnan R, Machado de Sousa SCOd Hunter KD. Altered Toll-like receptor expression and function in HPV-associated oropharyngeal carcinoma. Oncotarget. 2018;9(1):236248.

13. Rickinson AB. Co-infections, inflammation and oncogenesis: future directions for EBV research. Semin Cancer Biol. 2014;26:99-115.

14. Ruuskanen M, Leivo I, Minn H, Vahlberg T, Haglund C, Hagström J, et al. Expression of toll-like receptors in non-endemic nasopharyngeal carcinoma. BMC Cancer. 2019;19(1):624.

15. Aggarwal R, Misra S, Guleria C, Suri V, Mangat N, Sharma M, et al. Characterization of Toll-like receptor transcriptome in squamous cell carcinoma of cervix: a case-control study. Gynecol Oncol. 2015;138(2):358-62.

16. Rich AM, Hussaini HM, Parachuru VPB, and Seymour GJ. Toll-like receptors and cancer, particularly oral squamous cell carcinoma. Fronn Immunol. 2014;5:464.

17. Shi Y, Peng SL, Yang LF, Chen X, Tao YG, Cao Y. Co-infection of Epstein-Barr virus and human papillomavirus in human tumorigenesis. Chin J Cancer. 2016;35:16.

18. Sharma U, Singhal P, Bandil K, Patle R, Kumar A, Neyaz KD et al. Genetic variations of TLRs and their association with HPV/EBV, co-infection along with nicotine exposure in the development of premalignant/malignant lesions of the oral cavity in Indian population. Cancer Epidemiol. 2019;61:38-49.

19. Barros MR, de Oliveira THA, de Melo CML, Venuti Ad de Freitas AC. Viral Modulation of TLRs and Cytokines and the Related Immunotherapies for HPV-Associated Cancers. J Immunol Res. 2018.

20. Smith M, García-Martínez E, Pitter M, Fucikova J, Spisek R, Zitvogel Ld et al. Trial Watch: Toll-like receptor agonists in cancer immunotherapy. OncoImmunology. 2018;7(12):1-15. 